\title{
SATISFACCIÓN CON LA ALIMENTACIÓN EN PERSONAS MAPUCHES DE LA REGIÓN DE LA ARAUCANIA, CHILE
}

\author{
FOOD-RELATED LIFE SATISFACTION IN \\ MAPUCHES FROM THE ARAUCANÍA REGION, CHILE
}

\author{
Berta Schnettler M. (1), Marcos Mora G. (2), Horacio Miranda V. (1), \\ José Sepúlveda M. (3), Marianela Denegri C. (3), Germán Lobos A. (4)
}

(1) Departamento de Producción Agropecuaria, Facultad de Ciencias Agropecuarias y Forestales,

Universidad de La Frontera, Temuco, Chile.

(2) Departamento de Economía Agraria, Facultad de Ciencias Agronómicas,

Universidad de Chile, Santiago, Chile.

(3) Departamento de Psicología, Facultad de Educación y Humanidades.

Universidad de La Frontera, Temuco, Chile.

(4) Escuela de Ingeniería Comercial, Facultad de Ciencias Empresariales,

Universidad de Talca, Talca, Chile.

\begin{abstract}
In order to identify variables which have an influence on satisfaction with food-related life among mapuches a survey was applied to 400 mapuche subjects in the Araucanía Region. The questionnaire included the SWFL (Satisfaction with Food-related Life) scale. It was found that $39.5 \%$ were satisfied and $33.2 \%$ extremely satisfied with their food-related life. A multinomial probit model was proposed in which the dependent variable was foodsatisfaction, which resulted significant $(p<0.01)$ overall. The probability of high food-related satisfaction increases as fewer people live in the subject's household, more is spent on food, children express their opinion about foodstuffs, he/she consumes "catutos" (a mapuche food), aged 55 or over, not widowed, performs regular exercise, tries not to eat industrialized foods, but does not try to eat foods without additives. Food-related satisfaction in mapuche persons in the Araucanía Region is related to demographic, feeding and life-style variables.
\end{abstract}

Key words: food-related satisfaction; multinomial probit model; mapuche.

Este trabajo fue recibido el 18 de Enero de 2011 y aceptado para ser publicado el 15 de Enero de 2012.

\section{INTRODUCCIÓN}

Las personas están cada vez más conscientes de su autocuidado y buscan en el mercado aquellos productos que contribuyan a su salud y bienestar. Las carencias nutricionales globales ya no constituyen las prioridades de investigación. El interés actual se centra en la relación entre la alimentación y las enfermedades crónicas no transmisibles y en los efectos de la nutrición en las funciones cognitivas, inmunitarias, capacidad de trabajo y rendimiento deportivo (1).

El estudio y la medición de la satisfacción con la vida han generado gran interés en los últimos 15 años (2), pero se ha enfocado especialmente en medir los resultados del cuidado de la salud y de los servicios sociales
(3). Por este motivo, aún existe escasa investigación en torno a otras dimensiones de las necesidades de las personas. Dentro de éstas, la alimentación humana se ha convertido en uno de los temas principales de discusión en el ámbito científico, dado que además de la evidente relación entre la alimentación y la salud, el consumo de alimentos se encuentra mediado por muchos factores al margen de los estrictamente nutricionales.

La elección de los alimentos ha sido reconocida como un proceso que conlleva fuerzas psicológicas, sociales, culturales, económicas y biológicas (4). Los alimentos contribuyen al bienestar físico de las personas, son una importante fuente de placer y ocupan parte importante de su vida (5). Los alimentos cumplen una 
función utilitaria para el cuerpo, pero actúan como un producto hedónico y de construcción social que lleva a una auto definición de la persona (6). Los alimentos son preparados con la expectativa de ser compartidos y disfrutados en compañía (7), día tras día con la familia, y en los fines de semana con amigos (8).

A pesar de existir escasas investigaciones en relación con la satisfacción con los alimentos (9), se ha reportado que se relaciona con el estado de salud, los recursos disponibles, el apoyo familiar (10), la posibilidad de equilibrar el trabajo y la vida familiar (11) y con el consumo de ciertos alimentos (12).

En Chile, la encuesta de calidad de vida y salud considera en sus dimensiones los hábitos alimentarios, en cuanto al consumo de desayuno, sal y frecuencia de consumo de distintos alimentos (13).

La alimentación tiene un rol importante en los significados culturales, psicológicos, sociales y simbólicos, éstos últimos asociados a alimentos específicos, su forma de producción y preparación y los hábitos de alimentación (7). Los alimentos se asocian a la cultura y folklore de los pueblos (6). Los mapuche constituyen el grupo aborigen más grande de Chile y uno de los más grandes del continente (14). Diversos autores han sugerido y/o confirmado la existencia de un paulatino proceso de aculturación colectiva de los mapuche hacia la cultura chilena, incluyendo los hábitos alimentarios (15-17). Un estudio previo en la Región de La Araucanía considerando los grupos de alimentos que contemplan las Encuestas de Presupuestos Familiares, determinó que el gasto mensual en alimentos es menor en los hogares mapuche, respecto de los no mapuche (17). No obstante, se ha reportado que problemas de alimentación deficiente en niños mapuche, estarían más asociados a las condiciones sociales y no a la pertenencia étnica $(18,19)$.

Considerando que el bienestar material se asocia con pertenencias personales, incluyendo varios tipos de alimentos (20), el objetivo principal de esta investigación fue identificar variables que influyen en la satisfacción con la alimentación en personas pertenecientes a la etnia Mapuche residentes en la Región de La Araucanía, Chile. Considerando el proceso de aculturación alimentaria de los mapuche, se dio especial énfasis en determinar si el consumo de alimentos tradicionales de la etnia influye en la satisfacción con la alimentación.

\section{SUJETOS Y MÉTODOS}

Según datos del Censo de 2002 (21), de los 604.349 habitantes que declararon pertenecer a la etnia mapuche; 33,6\% habita en la Región de La Araucanía, es decir 203.061 personas, cifra que constituye el universo de la presente investigación. A partir de esto, se realizó una encuesta personal a una muestra de 400 personas de origen Mapuche, mayores de 18 años, que viven en la Región de La Araucanía, cuyo número se obtuvo mediante la fórmula de muestreo aleatorio simple para poblaciones no finitas ( $\mathrm{N}>100.000)$, considerando 95\% de confianza y $5 \%$ de error de estimación con $\mathrm{p}$ y q de $0,5(22)$.

Como instrumento de recogida de información se utilizó un cuestionario estructurado. La primera pregunta correspondió a una pregunta filtro destinada a controlar la composición de la muestra: ¿Ud. se considera una persona mapuche? Si la respuesta era positiva se procedía a aplicar el cuestionario, de lo contrario se agradecía la buena disposición y se daba por concluida la encuesta. Adicionalmente, la persona a encuestar debía cumplir con la condición de ser la encargada de realizar las compras de alimentos para su hogar. La segunda pregunta correspondió a la escala SWFL (Satisfaction with Food-related Life), que fue propuesta y probada por Grunert et al. (3) en ocho países europeos, mostrando adecuados niveles de consistencia interna y la existencia de una sola dimensión que agrupa los cinco ítems de la escala: 1. Los alimentos y comidas son elementos muy positivos en mi vida. 2. Yo estoy muy complacido con mi alimentación. 3. Mi vida en relación a los alimentos y comidas se acerca al ideal. 4. Respecto a los alimentos, mi condición de vida es excelente. 5. Los alimentos y comidas me proporcionan gran satisfacción en mi vida diaria. El encuestado debió responder su grado de acuerdo con cada una de estas afirmaciones mediante una escala tipo Likert de 6 niveles (1: completamente en desacuerdo, 6: completamente de acuerdo).

La SWFL fue probada en un estudio previo con una muestra de estudiantes, tanto de origen mapuche como no mapuche, de la Universidad de La Frontera, obteniéndose un adecuado nivel de consistencia interna ( $\alpha$ de Cronbach de 0,75) en la muestra estudiada (23).

En relación al consumo de alimentos, se consultó la cantidad aproximada de dinero que gastan mensualmente en este ítem. No se consideraron los alimentos que pudieran ser producidos para auto consumo en los sectores rurales, debido a que en el estudio realizado por Huaiquiñir (24) se determinó que una alta proporción de personas mapuche que residen en el campo comercializa los alimentos que producen o recolectan (hortalizas, huevos, aves de corral, corderos, frutos silvestres), con el objetivo de contar con mayores ingresos para comprar alimentos no mapuche, como arroz y fideos, entre otros. No obstante, para corroborar esto se consultó el lugar de compra u obtención de carne, abarrotes, frutas 
$\mathrm{y}$ verduras.

A continuación se consultó si consumen los siguientes alimentos y bebidas tradicionales mapuche: mudai (chicha elaborada con trigo machacado, pelado y hervido, fermentado varios días), tortilla de rescoldo (pan de harina de trigo cocido en ceniza caliente), catutos o mültrün (trigo sancochado molido, se le da forma alargada), pantrucas (masa de harina de trigo en forma cuadrada y huevo, cocido en caldo), charqui (carne deshidratada cubierta con sal y expuesta al sol), carne de caballo, piñón o pehuén (fruto de la Araucaria araucana. Comúnmente se come cocido), apol (hígado de cordero con ají, comino, sal, cilantro y aceite), ñachi (sangre de cordero con sal, cilantro, ají, limón y aceite), digüeñes (Cyttaria spp. Hongos parásitos que crecen sobre las ramas de algunos árboles nativos, especialmente el roble, también en coigües y otras especies del género Nothofagus), changles (Ramaria sp. Hongo de otoño e invierno que crece en el suelo de bosques esclerófilos o en las quebradas de bosque nativo caducifolio del sur de Chile), müllokin (a base de arvejas cocidas molidas, hechas pelotitas) y merkén (ají seco en vaina tostado, semilla de cilantro, sal y pimienta) (respuesta cerrada: sí, no). Si los encuestados respondían en forma afirmativa, se les consultó la frecuencia de consumo de estos alimentos (respuesta mixta: frecuentemente, sólo en fiestas familiares, sólo en celebraciones propias de la cultura mapuche, en la temporada en que el alimento está disponible, otra frecuencia de consumo). Se consultó si los niños opinan respecto a los alimentos que van a ser comprados y en la compra de qué tipo de alimentos opinan.

A continuación los encuestados debieron responder la escala de estilo de vida y alimentación propuesta por Sánchez et al. (25) que se compone de 15 ítems para ser contestada en forma de escala de Likert de cinco puntos (1: no lo hace; 5 : siempre). Los ítem de la escala son los siguientes: Controla la ingesta de sal. Practica una dieta vegetariana. Hace ejercicio con regularidad. Procura no comer alimentos industrializados. Come con frecuencia frutas y verduras. Come con moderación carne roja. Pertenece a una asociación de defensa de la naturaleza. Procura comer alimentos sin aditivos. Periódicamente chequea su salud voluntariamente. Procura reducir el estrés. Colabora con Organizaciones no Gubernamentales. Visita al dentista con regularidad. Procura llevar una vida ordenada y metódica. Procura equilibrar trabajo con vida privada. Lee las etiquetas de los productos.

Para comprobar que los ítems de las escalas SWFL y de estilo de vida y alimentación eran correctamente entendidas por personas de origen mapuche, previo a la aplicación de la encuesta se realizaron dos grupos focales con 10 participantes de origen mapuche cada uno, en los cuales se obtuvieron resultados satisfactorios al respecto.

Se incluyó una adaptación de la escala de aculturación mapuche desarrollada por Saiz et al. (15) y usada en un estudio previo con personas mapuche de la Región de La Araucanía (17). Finalmente, se incluyeron preguntas de clasificación sociodemográfica de los encuestados: género, edad, número de integrantes del grupo familiar; zona de residencia, estado civil, número de hijos o niños que viven en el hogar y sus edades, ocupación y estudios del jefe de hogar y, la tenencia de 10 bienes domésticos.

La encuesta se aplicó en lugares reconocidos por la afluencia de personas de origen mapuche en la capital de la Región de La Araucanía, la ciudad de Temuco, específicamente en las calles aledañas al terminal de buses rurales de la ciudad. Esto con el objetivo de acceder tanto a personas Mapuche residentes zonas urbanas, como en zonas rurales quienes viajan hasta Temuco para realizar compras y otros trámites. La encuesta se aplicó entre julio y agosto de 2009, posterior a la validación del cuestionario mediante un pretest con el $10 \%$ de la muestra. La realización del estudio fue aprobada por el Comité de Bioética de la Facultad de Ciencias Agropecuarias y Forestales de la Universidad de La Frontera. Los participantes firmaron consentimientos informados previo a su aplicación.

El análisis de los resultados fue realizado con el programa SPSS 16.0 en español para Windows. La extracción de factores de las escalas se realizó con análisis factorial de componentes principales, considerando autovalores mayores que 1 y rotación de factores Varimax con Kaiser (26). La consistencia interna de las escalas fue calculada usando el coeficiente $\alpha$ de Cronbach (26). Con el objeto de contrastar la influencia de distintas variables explicativas en la satisfacción con la alimentación en personas de etnia mapuche, se planteó un modelo probit multinomial ordinal, considerando como variable dependiente el nivel de satisfacción con la alimentación. El modelo probit ordinal es uno de los que se utilizan con más frecuencia para analizar los casos en que la variable dependiente refleja un orden (24). Como variables explicativas se incluyeron las respuestas obtenidas en el cuestionario aplicado, dejando finalmente las que resultaron estadísticamente significativas. Los parámetros del modelo probit fueron estimados por el método de máxima verosimilitud (27). Se utilizaron las siguientes medidas de la bondad de ajuste del modelo: $\mathrm{R}^{2}{ }_{\text {adj. }}$ de Nagelkerke (Pseudo- $\mathrm{R}^{2}$ ), - 2 log de verosimilitud y la prueba de Hosmer-Lemeshow. Para medir la significancia estadística de las variables explicativas se utilizó el estadístico Wald (27). La especificación de la variable dependiente y explicativas que resultaron significativas en el modelo se presentan en la tabla 1. 


\section{RESULTADOS}

La muestra estuvo compuesta principalmente por mujeres, menores de 55 años, casados o que viven en pareja, de familias formadas por tres a cuatro integrantes (Media: 3,98. Desv. típica: 1,733. Mínimo: 1. Máximo: 10), con hijos o niños de diferentes edades en el hogar, que residen en Temuco o en una pequeña ciudad de la Región de La Araucanía, con estudios básicos y medios incompletos, trabajadores independientes y empleados particulares, del nivel socioeconómico D (tabla 2). Cabe destacar que la mayor proporción de personas residentes en una pequeña ciudad de La Araucanía, provenían de las comunas de Victoria, Angol y Nueva Imperial. Aquellos que indicaron residir en un pueblo rural, principalmente provenían de la comuna de Chol-Chol, mientras que los residentes en el campo provenían mayoritariamente de los sectores rurales de las comunas de Carahue, Freire, Padre Las Casas y Puerto Saavedra.

El gasto promedio mensual en alimentos correspondió a \$75.377 (desv. típica: 39.251). Las personas

TABLA 1

Definición de variables dependiente y explicativas para el modelo probit multinomial de satisfacción con la propia alimentación en personas mapuche de la Región de La Araucanía, Chile. Agosto 2009.

\begin{tabular}{|c|c|c|c|c|}
\hline Modelo satisfacción alimentación & Dimensión & Categoría & $\begin{array}{l}\text { Código } \\
\text { (valores) }\end{array}$ & Definición (etiqueta) \\
\hline $\begin{array}{l}\text { Variable dependiente: } \\
\text { Satisfacción con la alimentación }\end{array}$ & Subjetiva & Multinomial & $\begin{array}{l}0 \\
1 \\
2 \\
3 \\
4\end{array}$ & $\begin{array}{l}\text { Extremadamente Insatisfecho } \\
\text { Insatisfecho } \\
\text { Medianamente Satisfecho } \\
\text { Satisfecho } \\
\text { Extremadamente Satisfecho }\end{array}$ \\
\hline $\begin{array}{l}\text { Variables explicativas: } \\
\text { Gasto en alimentación } \\
\text { Cantidad de integrantes }\end{array}$ & $\begin{array}{l}\text { Ingresos } \\
\text { Demográfica }\end{array}$ & $\begin{array}{l}\text { Continua } \\
\text { Continua }\end{array}$ & & $\begin{array}{l}\text { Gasto en alimentación } \\
\text { Integrantes del hogar }\end{array}$ \\
\hline Opinan niños de los alimentos & Percepción & Binaria & $\begin{array}{l}0 \\
1\end{array}$ & $\begin{array}{l}\mathrm{No} \\
\mathrm{Si}\end{array}$ \\
\hline Consume Catutos & Alimentos & Binaria & $\begin{array}{l}0 \\
1\end{array}$ & $\begin{array}{l}\text { No } \\
\mathrm{Si}\end{array}$ \\
\hline Edad del encuestado & Demográfica & Multinomial & $\begin{array}{l}0 \\
1 \\
2\end{array}$ & $\begin{array}{l}\text { Menos de } 35 \text { años } \\
\text { Entre } 35 \text { y } 54 \text { años } \\
55 \text { años o más }\end{array}$ \\
\hline Estado civil del encuestado & Demográfica & Multinomial & $\begin{array}{l}0 \\
1 \\
2 \\
3\end{array}$ & $\begin{array}{l}\text { Soltero(a) } \\
\text { Separado(a)/Divorciado(a) } \\
\text { Casado(a)/Vive en pareja } \\
\text { Viudo(a) }\end{array}$ \\
\hline Residencia del encuestado & Demográfica & Binaria & $\begin{array}{l}0 \\
1\end{array}$ & $\begin{array}{l}\text { Urbano } \\
\text { Rural }\end{array}$ \\
\hline Hace ejercicio con regularidad & Salud & Binaria & $\begin{array}{l}0 \\
1\end{array}$ & $\begin{array}{l}\text { No } \\
\mathrm{Si}\end{array}$ \\
\hline Consume alimentos industrializados & Salud & Binaria & $\begin{array}{l}0 \\
1\end{array}$ & $\begin{array}{l}\mathrm{No} \\
\mathrm{Si}\end{array}$ \\
\hline Consume alimentos sin aditivos & Salud & Binaria & $\begin{array}{l}0 \\
1\end{array}$ & $\begin{array}{l}\mathrm{No} \\
\mathrm{Si}\end{array}$ \\
\hline
\end{tabular}


TABLA 2

Descripción porcentual de la muestra. Región de La Araucanía, Chile. Agosto 2009.

\begin{tabular}{|c|c|c|}
\hline Muestra & & $\%$ \\
\hline Género & $\begin{array}{l}\text { Femenino } \\
\text { Masculino }\end{array}$ & $\begin{array}{l}59,5 \\
40,5\end{array}$ \\
\hline Edad & $\begin{array}{l}<35 \text { años } \\
35-54 \text { años } \\
55 \text { años y más }\end{array}$ & $\begin{array}{l}38,2 \\
42,2 \\
19,5\end{array}$ \\
\hline Estado civil & $\begin{array}{l}\text { Soltero(a) } \\
\text { Separado(a)/Divorciado(a) } \\
\text { Casado/Vive en pareja } \\
\text { Viudo(a) }\end{array}$ & $\begin{array}{r}28,2 \\
3,5 \\
61,0 \\
7,2\end{array}$ \\
\hline Tamaño grupo familiar & $\begin{array}{l}\text { 1-2 integrantes } \\
3-4 \text { integrantes } \\
5 \text { integrantes o más }\end{array}$ & $\begin{array}{l}22,5 \\
43,5 \\
34,0\end{array}$ \\
\hline Hijos y su edad & $\begin{array}{l}\text { Sin niños en el hogar } \\
\text { Niños o hijos menores de } 5 \text { años } \\
\text { Niños o hijos entre } 5 \text { y } 13 \text { años } \\
\text { Niños o hijos entre } 14 \text { y } 17 \text { años } \\
\text { Hijos mayores de edad }\end{array}$ & $\begin{array}{r}42,2 \\
20,8 \\
23,5 \\
12,2 \\
1,2\end{array}$ \\
\hline Zona de residencia & $\begin{array}{l}\text { Vive en la ciudad de Temuco } \\
\text { Vive en una pequeña ciudad } \\
\text { Vive en un pueblo rural } \\
\text { Vive en el campo }\end{array}$ & $\begin{array}{r}34,5 \\
29,0 \\
9,0 \\
27,5\end{array}$ \\
\hline Educación & $\begin{array}{l}\text { Sin estudios } \\
\text { Básica incompleta } \\
\text { Básica completa } \\
\text { Media incompleta } \\
\text { Media completa } \\
\text { Técnica incompleta } \\
\text { Técnica completa o universitaria incompleta } \\
\text { Universitaria completa o más }\end{array}$ & $\begin{array}{r}7,8 \\
25,5 \\
10,8 \\
12,0 \\
21,2 \\
3,8 \\
12,5 \\
6,5\end{array}$ \\
\hline Ocupación & $\begin{array}{l}\text { Trabajador por cuenta propia } \\
\text { Empresario } \\
\text { E. particular } \\
\text { E. público } \\
\text { Jubilado } \\
\text { Cesante } \\
\text { Otra }\end{array}$ & $\begin{array}{r}32,2 \\
2,8 \\
35,5 \\
10,8 \\
11,2 \\
3,0 \\
4,5\end{array}$ \\
\hline Nivel socioeconómico ${ }^{1}$ & $\begin{array}{l}\text { ABC1 (alto y medio alto) } \\
\text { C2 (medio-medio) } \\
\text { C3 (medio-bajo) } \\
\text { D (bajo) } \\
\text { E (muy bajo) }\end{array}$ & $\begin{array}{r}9,5 \\
17,2 \\
21,8 \\
32,5 \\
19,0\end{array}$ \\
\hline
\end{tabular}


encuestadas indicaron obtener la carne principalmente en supermercados $(44,1 \%)$, carnicerías $(30,1 \%)$ o de producción propia $(16,6 \%)$; los abarrotes en supermercados $(76 \%)$ y en almacenes de barrio $(13,6 \%)$; las frutas y verduras en ferias libres $(37,6 \%)$, supermercados $(18,4 \%)$ o de producción propia $(13,8 \%)$. El 46,1\% de los encuestados declaró que los niños opinan sobre los alimentos que se compran, pero la mayoría opina respecto a la compra de golosinas y cereales para el desayuno $(36,3 \%)$. Sólo en 5,2\% de los casos los niños opinan respecto a la elección de alimentos para preparar comidas y en el $12,6 \%$ opinan en la elección de ambos tipos de alimentos.

Los resultados de la escala de aculturación permitieron identificar a la mayor proporción de la muestra como aculturados $(35,5 \%)$ o biculturales $(61,2 \%)$, con un coeficiente $\alpha$ Cronbach $(0,765)$ que indica que la escala es fiable (26). La escala de estilo de vida y alimentación (25) presentó un $\alpha$ de Cronbach de 0,803 y dos factores: Salud (27,2\% varianza explicada) y Ecología $(12,3 \%)$, con cuatro estilos de vida: no ecológicos y despreocupados por la salud $(29,0 \%)$, preocupados por la ecología $(22,5 \%)$, preocupados por la salud $(29,0 \%)$ y preocupados por la salud y la ecología $(19,5)$. Respecto a la escala de satisfacción con la propia alimentación (SWFL), mediante análisis factorial de componentes principales se obtuvo la existencia de un solo factor para el total de los ítems con el $67,5 \%$ de la varianza explicada. El coeficiente $\alpha$ Cronbach obtenido $(0,878)$ indica un adecuado nivel de consistencia interna y permite concluir que se trata de una escala fiable (26). Predominaron los sujetos satisfechos $(39,5 \%)$ y extremadamente satisfechos $(33,2 \%)$ con su alimentación (tabla 3).

La tabla 4 presenta la proporción de personas encuestadas que indicó consumir los alimentos tradicionales consultados y su frecuencia de consumo. De los alimentos más consumidos destacan los digüeñes, pantrucas, tortillas de rescoldo y piñones, mientras que los menos consumidos fueron el apol y el müllokin. Paralelamente, destacan las tortillas de rescoldo, pantrucas y el merkén por ser los alimentos consumidos en mayor proporción frecuentemente. El mudai, ñachi y los catutos son consumidos en porcentaje superior asociados a celebraciones culturales propias de la etnia mapuche. El ñachi fue el único alimento tradicional que presentó un mayor porcentaje de consumo en fiestas familiares. Los piñones, digüeñes y changles son consumidos principalmente en la temporada de disponibilidad. En las familias con niños, en 45,6\% de los casos los niños no opinan respecto de los alimentos que serán preparados.

El modelo probit multinomial estimado resultó significativo en su conjunto ( $\mathrm{p} \leq 0,01)$, lo cual significa que el modelo es un buen predictor según las pruebas $\mathrm{R}_{\text {adj. }}^{2}$ de Nagelkerke, -2 log de versosimilitud y HosmerLemeshow (tabla 5). De todas las variables probadas en el modelo resultaron significativas $(\mathrm{p}<0,01)$ si los niños opinan en la elección de los alimentos a preparar, si la persona tiene menos de 35 años respecto de aquellos que tienen 55 años o más y, si la persona acostumbra a hacer ejercicio regularmente. Además, resultaron significativas $(\mathrm{p}<0,05)$ la cantidad de integrantes del grupo familiar, si la persona tiene entre 35 y 54 años respecto de aquellos con 55 años o más, si la persona es separada o divorciada o si está casada o vive en pareja respecto de los encuestados viudos, la zona de residencia y el consumo de alimentos industrializados. Las variables gasto en alimentación, consumo de catutos, si la persona está soltera respecto de los viudos y el consumo de

\section{TABLA 3}

\section{Descripción porcentual de la muestra (\%) según el grado de satisfacción con su alimentación en la Región de La Araucanía, Chile. Agosto 2009.}

Satisfacción con la alimentación

$\begin{array}{lc}\text { Extremadamente insatisfecho } & 0,5 \\ \text { Insatisfecho } & 5,0 \\ \text { Medianamente satisfecho } & 21,8 \\ \text { Satisfecho } & 39,5 \\ \text { Extremadamente satisfecho } & 33,2 \\ \text { Varianza explicada (\%) } & 67,500 \\ \alpha \text { Cronbach } & 0,878\end{array}$


alimentos sin aditivos, resultaron significativas al nivel $\mathrm{p}<0,1$. Considerando el signo de los coeficientes y las categorías de comparación del modelo probit (tabla 5), se concluye que:

- Es posible esperar una menor probabilidad de alta satisfacción con la alimentación mientras mayor sea la cantidad de integrantes del hogar $(\beta=-0,265)$.

- $\quad$ Es posible esperar una mayor probabilidad de alta satisfacción con la alimentación en la medida que aumente el gasto en alimentos $(\beta=7,81 \mathrm{E}-0,006)$.

- Es posible esperar una reducción en la probabilidad de alta satisfacción con la alimentación si los niños no opinan respecto a la compra de alimentos ( $\beta=-0,879)$, en comparación con las personas que si reciben la opinión de los niños que viven en el hogar.

- Es posible esperar una reducción en la probabilidad de alta satisfacción con la alimentación si la persona no consume catutos $(\beta=-0,521)$, en comparación con las personas que si consumen este alimento tradicional mapuche.

- Si el encuestado posee entre 35 y 54 años disminuye la probabilidad de alta satisfacción con la alimentación con respecto de personas con 55 años o más $(\beta=-1,457)$, siendo esta última la categoría de comparación. La misma conclusión se obtiene para las personas menores de 35 años $(\beta=-2,007)$.

- $\quad$ Si el encuestado es soltero aumenta la probabilidad de alta satisfacción con la alimentación con respecto de personas viudas $(\beta=2,057)$, siendo esta última la categoría de comparación. La misma conclusión se obtiene para las personas separados o divorciadas $(\beta=3,608)$ y casadas o que viven en pareja $(\beta=$ $3,608)$.

- Es posible esperar una reducción en la probabilidad de alta satisfacción con la alimentación si la persona vive en un sector urbano $(\beta=-0,757)$, en comparación con las personas que residen en sectores rurales.

- Si el encuestado declara no hacer ejercicio con regularidad disminuye la probabilidad de alta satisfacción con la alimentación $(\beta=-1,806)$, con respecto a las personas que si procuran ejercitarse.

- Es posible esperar una reducción en la probabilidad de alta satisfacción con la alimentación si la persona no procura evitar el consumo de alimentos industrializados $(\beta=-0,911)$, en comparación con la persona que indicó procurar no comer alimentos industrializados.

- La probabilidad de alta satisfacción con la alimentación aumenta si la persona no procura consumir alimentos sin aditivos $(\beta=0,753)$, en contraste con la persona que si lo hace.

\section{TABLA 4}

Consumo y frecuencia de consumo de alimentos tradicionales $(\%)$ en personas mapuche de la Región de La Araucanía, Chile. Agosto 2009.

\begin{tabular}{|lcccccc|}
\hline Alimento & Consumo & \multicolumn{3}{c}{ Frecuencia de consumo } \\
& & $\begin{array}{c}\text { Frecuen- } \\
\text { temente }\end{array}$ & $\begin{array}{c}\text { Fiestas } \\
\text { familiares }\end{array}$ & $\begin{array}{c}\text { Fiestas } \\
\text { culturales }\end{array}$ & $\begin{array}{c}\text { Temporada de } \\
\text { disponibilidad }\end{array}$ & Otra \\
\hline Mudai & 52,0 & 13,0 & 7,2 & 29,0 & 2,0 & 0,8 \\
Tortilla de rescoldo & 82,2 & 75,4 & 4,9 & 9,4 & 2,1 & 8,2 \\
Catutos o Mültrun & 52,0 & 21,8 & 5,8 & 19,0 & 1,2 & 4,2 \\
Pantrucas & 80,0 & 73,2 & 1,0 & 3,0 & 0,5 & 2,2 \\
Charqui & 52,5 & 28,2 & 7,5 & 10,0 & 3,5 & 3,2 \\
Carne de caballo & 68,8 & 43,5 & 9,5 & 11,5 & 1,5 & 2,8 \\
Piñones & 84,2 & 1,0 & 0,2 & 1,5 & 81,2 & 0,2 \\
Apol & 26,5 & 3,0 & 8,5 & 12,0 & 2,0 & 1,0 \\
Nachi & 52,2 & 4,2 & 17,8 & 23,8 & 5,8 & 0,8 \\
Digüieñes & 89,2 & 1,2 & 0,5 & 1,2 & 86,2 & 0 \\
Changle & 56,8 & 1,8 & 2,0 & 4,8 & 48,2 & 0 \\
Müllokin & 30,2 & 11,5 & 6,0 & 11,8 & 0,8 & 0,2 \\
Merkén & 72,0 & 66,0 & 1,2 & 2,0 & 0,8 & 2,0 \\
\hline
\end{tabular}




\section{TABLA 5}

\section{Resultados del modelo probit multinomial de satisfacción con la propia alimentación en personas mapuche de la Región de La Araucanía, Chile. Agosto, 2009.}

\begin{tabular}{|c|c|c|c|c|c|}
\hline \multirow[t]{2}{*}{$\begin{array}{l}\text { Variable dependiente: Grado de } \\
\text { satisfacción con la propia alimentación }\end{array}$} & \multirow[t]{2}{*}{ Beta } & \multirow[t]{2}{*}{ Wald } & \multirow[t]{2}{*}{ Sig. } & \multicolumn{2}{|c|}{$\begin{array}{l}\text { 95\% Límites de intervalo } \\
\text { de confianza para Beta }\end{array}$} \\
\hline & & & & Inferior & Superior \\
\hline \multicolumn{6}{|l|}{ Umbral } \\
\hline$\gamma_{0} \quad$ Extremadamente Insatisfecho & $* * *-8,658$ & & 0,000 & $-12,574$ & $-4,741$ \\
\hline$\gamma_{1}$ Insatisfecho & $* * *-6,200$ & & 0,000 & $-9,648$ & $-2,752$ \\
\hline$\gamma_{2}$ Medianamente Satisfecho & $* *-4,103$ & & 0,018 & $-7,493$ & $-0,712$ \\
\hline$\gamma_{3}$ Satisfecho & $-1,654$ & & 0,332 & $-4,998$ & 1,690 \\
\hline Cantidad de integrantes & $* *_{-} 0,265$ & 5,784 & 0,016 & $-0,481$ & $-0,049$ \\
\hline Gasto en alimentación & $* 7,81 \mathrm{E}-0,006$ & 3,534 & 0,060 & $-3,32 \mathrm{E}-007$ & $1,59 \mathrm{E}-005$ \\
\hline \multicolumn{6}{|l|}{ Opinan niños de los alimentos } \\
\hline No & $* * *-0,879$ & 8,379 & 0,004 & $-1,475$ & $-0,284$ \\
\hline $\mathrm{Si}$ & $0^{\mathrm{C}}$ & . & & & \\
\hline \multicolumn{6}{|l|}{ Consume Catutos } \\
\hline No & $*_{-0,521}$ & 2,929 & 0,087 & $-1,117$ & 0,076 \\
\hline $\mathrm{Si}$ & $0^{\mathrm{C}}$ & . & & & \\
\hline \multicolumn{6}{|l|}{ Edad del encuestado } \\
\hline Menos de 35 años & $* * *-2,007$ & 8,887 & 0,003 & $-3,327$ & $-0,688$ \\
\hline Entre 35 y 54 años & $* *-1,457$ & 5,664 & 0,017 & $-2,657$ & $-0,257$ \\
\hline 55 años o más & $0^{\mathrm{C}}$ & . & & & . \\
\hline \multicolumn{6}{|l|}{ Estado civil del encuestado } \\
\hline Soltero(a) & $* 2,057$ & 3,337 & 0,068 & $-0,150$ & 4,264 \\
\hline Separado(a)/Divorciado(a) & $* * 3,608$ & 6,381 & 0,012 & 0,809 & 6,407 \\
\hline Casado(a)/Vive en pareja & $* * 2,372$ & 5,022 & 0,025 & 0,298 & 4,447 \\
\hline Viudo(a) & $0^{\mathrm{C}}$ & . & & & , \\
\hline \multicolumn{6}{|l|}{ Residencia del encuestado } \\
\hline Urbano & $* *_{-} 0,757$ & 4,644 & 0,031 & $-1,446$ & $-0,069$ \\
\hline Rural & $0^{\mathrm{C}}$ & . & & & . \\
\hline \multicolumn{6}{|l|}{ Hace ejercicio con regularidad } \\
\hline No & $* * *-1,806$ & 13,862 & 0,000 & $-2,757$ & $-0,855$ \\
\hline $\mathrm{Si}$ & $0^{\mathrm{C}}$ & . & & & \\
\hline \multicolumn{6}{|l|}{ No come alimentos industrializados } \\
\hline No & $* *_{-} 0,911$ & 5,368 & 0,020 & $-1,681$ & $-0,142$ \\
\hline $\mathrm{Si}$ & $0^{\mathrm{C}}$ & . & & & \\
\hline \multicolumn{6}{|l|}{ Consume alimentos sin aditivos } \\
\hline No & $* 0,753$ & 3,382 & 0,066 & $-0,050$ & 1,556 \\
\hline $\mathrm{Si}$ & $0^{\mathrm{C}}$ & . & & & , \\
\hline $\mathrm{R}_{\text {adj. }}^{2}\left(\text { Pseudo- } \mathrm{R}^{2}\right)^{\mathrm{d}}$ & \multicolumn{5}{|c|}{$* * * 0,346$} \\
\hline $\begin{array}{l}-2 \log \text { de verosimilitud }(\mathrm{LR})^{\mathrm{e}}, \\
\text { modelo final }\end{array}$ & $* * * 423,655$ & $\chi^{2}=79,016$ & li & $\begin{array}{l}\text { os de } \\
d=26\end{array}$ & Sig. $=0,000$ \\
\hline $\begin{array}{l}\text { "Variables y modelos significativos al nivel } * \mathrm{P}<0,10 \text {; } \\
\text { y las pruebas Pseudo- } \mathrm{R}^{2},-2 \text { log de verosimilitud y de } \\
\text { Los valores entre paréntesis corresponden al estadísti } \\
\text { "Categoría de comparación. Este parámetro se estable } \\
\text { 'Se usó el R2 de Nagelkerke como proxy del coeficie } \\
\text { Prueba LR (Likehood Ratio) o "logaritmo del cocier } \\
\text { La prueba LR permite medir el peso de las variables }\end{array}$ & $\begin{array}{l}\text { P }<<0,05 ; * * * \mathrm{P}<0,011 \\
\text { losmer-Lemeshow pa } \\
\text { o de Wald. } \\
\text { e en } 0 \text { porque es redur } \\
\text { te de determinación ( } \\
\text { de verosimilitud" pa } \\
\text { xplicativas en la veros }\end{array}$ & $\begin{array}{l}\text { sados en el estadíst } \\
\text { bondad del ajuste } \\
\text { ante. } \\
\text { udo-R2) en el Moc } \\
\text { medir bondad de a } \\
\text { ilitud del modelo } \mathrm{f}\end{array}$ & $\begin{array}{l}\text { tico de W } \\
\text { de los mo } \\
\text { delo Prob } \\
\text { ajuste en }\end{array}$ & lo Probit. & le variables \\
\hline
\end{tabular}




\section{DISCUSIÓN}

Un primer elemento que se destaca de los resultados de este estudio son los altos niveles de satisfacción con la alimentación que declaran sentir los participantes en la encuesta. No obstante, los resultados del presente estudio son levemente inferiores a los obtenidos en una muestra de 400 personas de origen mapuche en la Región Metropolitana, donde 40,5\% estuvo satisfecho y $41,0 \%$ extremadamente satisfecho con su alimentación (28). También fueron levemente inferiores a los resultados obtenidos en una muestra de estudiantes de la Universidad de La Frontera, en la cual 46,4\% estuvo satisfecho y $35,7 \%$ extremadamente satisfecho con su alimentación (23). No obstante, es destacable que al considerar sólo las categorías "satisfecho" e "insatisfecho" en dicho estudio, se encontró una mayor proporción de participantes que declararon pertenecer a la etnia mapuche y que se encontraban insatisfechos con su alimentación. Paralelamente, en el subgrupo mapuche, la insatisfacción con la propia alimentación estuvo asociada a estudiantes pertenecientes a hogares en que la persona que aporta el principal ingreso económico es de género femenino, mientras que en el subgrupo de estudiantes no mapuche surge el nivel de estudios de quien aporta el principal ingreso económico en el hogar del encuestado, como variable asociada a la satisfacción con la alimentación. Por otra parte, en un estudio realizado con una muestra mayoritariamente no mapuche en la ciudad de Temuco, se obtuvo que el 53,0\% estuvo satisfecho y el 36,5\% extremadamente satisfecho con su alimentación (29). Frente a estos antecedentes, es posible sugerir que el nivel de satisfacción con la alimentación se asocia a múltiples variables, como el acceso a los estudios, la región de residencia, el género de la persona que aporta el principal ingreso al hogar, entre otras, dentro de las cuales también se encuentra el origen étnico. Si bien se evidencia un alto nivel de bienestar subjetivo en relación a los hábitos alimentarios, también podría constituirse en una dificultad para eventuales estrategias de intervención en esta área.

Los resultados del modelo probit planteado permiten dar cuenta de una relación entre la satisfacción con la alimentación y las características demográficas de la persona mapuche, aspectos asociados al consumo de alimentos y su estilo de vida.

Respecto de las características demográficas, llama la atención la disminución de la probabilidad de una alta satisfacción con la alimentación en la medida que aumenta el número de integrantes del hogar, debido a que la familia constituye una de las principales fuentes de satisfacción para las personas (30). Sin embargo, existe evidencia de que padres que trabajan y poseen familias numerosas presentan menores niveles de satisfacción con la alimentación, debido a que se sienten abrumados por las múltiples demandas familiares (11).

Asimismo, la disminución de la probabilidad de alta satisfacción con la alimentación si los niños no opinan respecto a la compra de alimentos, concordaría con la menor satisfacción que experimentan padres que no reciben ayuda de sus hijos en la obtención y preparación de alimentos (11). Sin embargo, debido a que la mayor proporción de niños opina respecto a la compra de golosinas y cereales para el desayuno ( $48,9 \%$ en conjunto), es posible sugerir que en los padres de niños que opinan sobre la compra de estos productos, aumenta la satisfacción con la alimentación en el sentido de poder satisfacer las necesidades relacionadas con el placer hedónico que experimentan sus hijos al comer golosinas y cereales para el desayuno.

La menor probabilidad de satisfacción con la alimentación en personas menores de 35 años y con edades entre 35 y 54 años con respecto de personas con 55 años o más, sería congruente con el aumento de la satisfacción con la vida conforme aumenta la edad, debido a que las personas a medida que envejecen van ajustando sus metas según sus posibilidades (30). Posibles causas que esto, serían la mayor preocupación por el cuidado de la salud en el consumo de alimentos en la medida que avanza la edad de las personas (31) y el aumento en la satisfacción con la apariencia física a mayor edad (32). No obstante lo anterior, las causas de la relación entre la edad y la satisfacción con la alimentación deberán ser profundizadas en futuras investigaciones. En relación a la menor satisfacción con la alimentación en personas viudas respecto del resto de los estados civiles, se ha sugerido que el matrimonio y la familia actuarían como un soporte protector emocional e instrumental (30). Considerando que los alimentos se disfrutan junto a los amigos y la familia $(7,8)$, es posible esperar que la menor satisfacción en las personas viudas se relacione con el alimentarse en solitario muchas veces sobre todo a mayor edad, como es el caso de la muestra en estudio en que se obtuvo una significativa superior proporción de viudos(as) de 55 años o más $(\mathrm{p}=0,000)$. Esto concordaría con la mayor satisfacción con la alimentación en personas de la tercera edad en Europa, si cuentan con la compañía de familiares para la preparación y consumo de alimentos (10).

La disminución de la probabilidad de una alta satisfacción con la alimentación si la persona mapuche reside en una zona urbana resulta preocupante, debido la alta concentración de población mapuche en sectores urbanos (14), producto de los marcados procesos de migración rural-urbana en búsqueda de mejores con- 
diciones económicas y de vida (33), pero que a la luz de los resultados pareciera generar el efecto opuesto. Este resultado se asociaría a la posibilidad que tienen los mapuche que residen en el campo, de obtener ingresos a partir de la comercialización de productos agrícolas (hortalizas como lechugas y cilantro y frutas de temporada), pecuarios (corderos, queso, huevos) y de recolección (murtillas, nalcas), que permiten a esta etnia comprar los productos que no se tienen o no se producen en el campo, como aceite, azúcar, yerba mate, fideos y arroz $(24,34)$.

En relación al consumo de alimentos, el aumento de la probabilidad de una alta satisfacción con la alimentación en la medida que es posible gastar un monto superior en alimentos, se asocia a la posibilidad de acceder a una alimentación de mayor calidad, variedad y más saludable (35). Esto es congruente con el bajo monto gastado en alimentos en la muestra en estudio, el cual representa el 49,1\% del valor promedio obtenido en la VI Encuesta de Presupuesto Familiares del INE (36) en las capitales regionales ( $\$ 153.363$ promedio), concordando además con estudios que relacionan los problemas de alimentación deficiente en mapuches con sus condiciones socioeconómicas $(18,19)$. A pesar que la alimentación constituye una parte fundamental de la identidad cultural de las sociedades (6), en este estudio se obtuvo aumento de la probabilidad de alta satisfacción con la vida con el consumo de sólo un alimento tradicional mapuche, los catutos, los cuales destacaron por su mayor frecuencia de consumo en celebraciones culturales mapuche. Esto difiere de lo encontrado en personas mapuche residentes en la Región Metropolitana, en que la satisfacción con la alimentación se incrementa mediante el consumo de alimentos tradicionales de fácil accesibilidad y preparación, y no con aquellos relacionados a las ceremonias rituales (28). A la vez difiere de los resultados obtenidos por Araneda et al. (37) en un estudio sobre los hábitos de consumo de alimentos autóctonos en adolescentes (mujeres) mapuche de la Región de La Araucanía. En dicha investigación se obtuvo que alrededor del $80 \%$ consume alimentos que conllevan una rápida preparación antes de su consumo; como son las castañas y piñones, que corresponden a frutos cocidos en agua.

Lo anterior, junto a que no se obtuvo relación entre la satisfacción con la alimentación y el grado de aculturación, se relacionarían con la aculturación alimentaria de los mapuche en la Región de La Araucanía (17), puesto que las preferencias hacia los alimentos constituyen un importante indicador de identificación étnica (38). Esto se refuerza al considerar que en el presente estudio, se obtuvo que una importante proporción encuestados que adquiere alimentos como la carne, abarrotes, frutas y verduras en supermercados y ferias libres, siendo las mujeres las que en mayor proporción realizan las compras de alimentos, de forma similar a las costumbres propias de la cultura chilena (39).

Si bien la satisfacción con la alimentación no se relacionó directamente con los resultados de la escala de estilo de vida y alimentación (22), si se obtuvo relación con tres de los ítems que la componen. Es destacable la reducción en la probabilidad de alta satisfacción con la alimentación si la persona no hace ejercicio con regularidad y, si no procura no consumir alimentos industrializados, lo que indica que la satisfacción con la alimentación se relacionaría con la búsqueda de una alimentación natural y un estilo de vida no sedentario. No obstante lo anterior, estos resultados se contraponen al aumento de la probabilidad de alta satisfacción con la alimentación si la persona no procura consumir alimentos sin aditivos, aspecto que pudiendo estar asociado al proceso de aculturación alimentaria de la etnia mapuche, deberá ser profundizado en futuras investigaciones.

\section{RESUMEN}

Con el objetivo de identificar variables que influyen en la satisfacción con la alimentación en personas mapuche, se aplicó una encuesta a 400 sujetos de esta etnia en la Región de La Araucanía. El cuestionario incluyó la escala SWFL (Satisfaction with Food-related Life). Se obtuvo que el 39,5\% está satisfecho y el 33,2\% extremadamente satisfecho con su alimentación. Se planteó un modelo probit multinomial en que la variable dependiente fue la satisfacción con la alimentación, el cual resultó significativo $(\mathrm{p}<0,01)$ en su conjunto. La probabilidad de una alta satisfacción con la alimentación aumenta en la medida que en el hogar del individuo viven menos personas, se gasta más en alimentación, los niños opinan respecto a los alimentos, consume catutos, tiene 55 años de edad o más, no es viudo, realiza regularmente ejercicio, procura no comer alimentos industrializados, pero no procura comer alimentos sin aditivos. La satisfacción con la alimentación en mapuche de La Araucanía se relaciona con variables demográficas, alimentación $\mathrm{y}$ estilo de vida.

Palabras clave: satisfacción con la alimentación; modelo probit multinomial, mapuche.

Agradecimientos: Los resultados presentados corresponden al Proyecto Fondecyt 1080146 y Fondecyt 1100611 .

Dirigir la correspondencia a:

Profesora

Berta Schnettler M 
Departamento de Producción Agropecuaria

Facultad de Ciencias Agropecuarias y Forestales

Universidad de La Frontera

Casilla 54-D

Temuco, Chile

56-45-325655/56-45-325458

E-mail:bschnett@ufro.cl

\section{BIBLIOGRAFÍA}

1. Araya H, Lutz M. Alimentos funcionales y saludables. Rev Chil Nutr 2003; 30(1): 8-14.

2. Moons P, Budts W, De Geest S. Critique on the conceptualization of quality of life: A review and evaluation of different conceptual approaches. Int J Nurs Stud 2006; 43(7): 891-901.

3. Grunert K, Dean D, Raats M, Nielsen N, Lumbers M. A measure of satisfaction with food-related life. Appetite 2007; 49(2): 486-93.

4. Bisogni C, Connors M, Devine C, Sobal J. Who We Are and How We Eat: A Qualitative Study of Identities in Food Choice. J Nutr Educ Behav 2002; 34(3): 128-39.

5. Rozin P, Fischler C, Imada S, Sarubin A, Wrzesniewski A. Attitudes to food and the role of food in life in the USA, Japan, Flemish Belgium and France: possible implications for the diet-health debate. Appetite 1999; 33(2): 163-80.

6. Hausman A. Hedonistic rationality: the duality of food consumption. Adv Consumer Res 2005; 32: 404-5.

7. Kniazeva K, Venkatesh A. Food for thought: A study of food consumption in postmodern US culture. Journal of Consumer Behavior 2007; 6(6): 419-35.

8. Casotti L. He who eats alone will die alone? An exploratory study of the meanings of the food of celebration. Lat Am Bus Rev 2005; 6(4): 69-84.

9. Veenhoven R. Effects of happiness on physical health and the consequences for preventive health care. Journal of Happiness Studies 2008; 9: 449-69.

10. Dean M, Grunert K, Raats M, Nielsen NA, Lumbers M, Food in Later Life Team. The impact of personal resources and their goal relevance on satisfaction with food-related life among the elderly. Appetite 2008; 50(2-3): 308-15.

11. Blake C, Devine C, Wethington E, Jastran M, Farrell T, Bisogni, C. Employed parents' satisfaction with food-choice coping strategies. Influence of gender and structure. Appetite 2009; 52(3): 711-9.

12. Ventegodt S. Livskvalitet og omsteandigheder tidligt I livet. Forknigscenter for Livskvalitet, Kobenhaven, Denmark, 1997.

13. Ministerio de Salud Chile. 2007. II encuesta de calidad de vida y salud chile 2006. Informe de resultados total nacional Disponibleen. http.//epi minsal_llepi/htm//sdesalud/calidaddevida2006 Informe\%20Final\%20Encuesta\%20 de\%20Calidad\%20de\%20Vida\%20y\%20Salud\%202006.pdf [consultado en noviembre 2010].

14. MIDEPLAN. 2005. Encuesta de caracterización socioeconómica nacional y pqhación indígena CASEN 2003 Disponible en htp.//www mideplan.cl/casen/pdf/casen2003poblacionindigena.pdf [consultado en mayo 2007].

15. Saiz JL, Cornejo A, Fuchslocher V, Holzapfel J, Scheel M. La escala de aculturación mapuche: diferenciando la dirección aculturativa de indígenas chilenos. Rev Psicol Social Personalidad 1998; 14(1):15-28.

16. Carrasco E, Pérez F, Bárbara B, Albala C, Santos JL, Larenas G, et al. Prevalencia de diabetes tipo 2 y obesidad en dos poblaciones aborígenes de Chile en ambiente urbano. Rev Méd Chil 2004; 132(10): 1189-97.

17. Schnettler B, Huaiquiñir V, Mora M, Miranda H, Sepúlveda J, Denegri, M. Diferencias étnicas y de aculturación en el consumo de alimentos en la Región de La Araucanía, Chile. Rev Chil Nutr 2010; 37(1): 31-40.

18. Amigo H, Bustos P, Erazo M, Radrigán ME. Comparación del estado nutricional de escolares indígenas y no indígenas. Rev Méd Chil 1999; 127 (8): 903-10.

19. García V, Amigo R, Bustos P. Ingesta alimentaria calórica en escolares chilenos de procedencia indígena y no indígena de diferente vulnerabilidad social. Arch Latinoam Nutr 2002; 52(4): 12-27.

20. Bell V. Consuming geographies: we are what we eat. Routledge, London, 1997.

21. INE. Censo 2002. Resultados Volumen I: Población; País - Región. Instituto Nacional de Estadísticas, Gobierno de Chile, Santiago, Chile, 2003.

22. Fernández A. Investigación y técnicas de mercado. Primera edición. Esic, Madrid, 2002.

23. Schnettler B, Miranda H, Sepúlveda J, Denegri M. Satisfacción con la Alimentación y la Vida, un Estudio Exploratorio en Estudiantes de la Universidad de La Frontera, Temuco- Chile. Psicol Sociedad 2011; 23 (2): 426-35.

24. Huaiquiñir, V. Características de los hábitos de consumo de alimentos en consumidores de distinta etnia en la Región de La Araucanía. Tesis Universidad de La Frontera, 2010.

25. Sánchez M, Grande I, Gil JM, Rodríguez A. Evaluación del potencial de mercado de los productos de 
agricultura ecológica. Revista de Investigación de Marketing ESIC 1998; Marzo: 135-50.

26. Pérez C. Técnicas estadísticas con SPSS 12. Aplicaciones al análisis de datos. Prentice Hall, Madrid, 2005.

27. Greene WH.Análisis econométrico. $3^{\mathrm{a}}$ ed.PrenticeHall, Madrid, 2008.

28. Schnettler B, Miranda H, Sepúlveda J, Denegri M, Mora M, Lobos G. Satisfacción con la alimentación en personas Mapuche en la Región Metropolitana de Santiago, Chile. Arch Latinoam Nutr 2011; 61(2): 172-82.

29. Schnettler B, Shene C, Rubilar M, Miranda H, Sepúlveda J, Denegri M, Lobos G. Aceptación hacia yogurt con diferentes ingredientes funcionales en consumidores de supermercados del sur de Chile. Arch Latinoam Nutr 2010; 60(4): 380-90.

30. Moyano E, Ramos N. Bienestar subjetivo: midiendo satisfacción vital, felicidad y salud en población chilena de la Región Maule. Universum 2007; 22(2): 177-93.

31. Beardsworth A, Bryman A, Keil T, Goode J, Haslam C. Lancashire, E. Women, men and food: the significance of gender for nutritional attitudes and choices. Br Food J 2002; 107(7): 470-91.

32. Loland NW. The aging body: attitudes toward bodily appearance among physically active and inactive woman and men of different ages. J Aging Phys Act 2000; 8(3): 197-213.

33. Grebe M. Creencia e identidad en la cultura Mapu- che: rewe, kultrun y ngillatue. Lengua y Literatura Mapuche 2000; 9: 273-88.

34. Carrasco, N. Antropología de los problemas alimentarios contemporáneos. Etnografía de la intervención alimentaria en la Región de La Araucanía, Chile. Tesis doctoral. Universitàt Autónoma de Barcelona Facultad de Lletres Departament d'Antropología Social i Cultural. Barcelona, España. 2004.

35. French S, Wall M, Mitchell N. Household income differences in food sources and food items purchased. Int J Behav Nutr Phys Act 2010; 7: 77.

36. INE. Resultados Encuestas de Presupuestos familiares Nov 2006-oct 2007 Disponible en. http./ www ine cl/canales/chile estadistico/encuestas presupuestos_familiares/2008/resultados_EPF_ 2006_2007_080708a.pdf][consultado en diciembre 2008].

37. Araneda J, Amigo H, Bustos P. Características alimentarias de adolescentes chilenas indígenas y no indígenas. Arch Latinoam Nutr 2010; 60(1): 30-5

38. Laroche M, Kim Ch, Tomuik M, Belisle D. Similarities in Italian and Greek multidimensional ethnic identity: some implications for food consumption. Can J Adm Sci 2005; 22(2): 143-67.

39. Schnettler B, Vidal R, Silva R, Vallejos L, Sepúlveda $\mathrm{N}$. Consumer Willingness to Pay for Beef Meat in a Developing Country: The Effect of Information Regarding Country of Origin, Price and Animal Handling Prior to Slaughter. Food Qual Prefer 2009; 20: 156-65. 